\title{
UK Renal Registry 15th Annual Report: Appendix A The UK Renal Registry Statement of Purpose
}

1. Executive summary

2. Introduction

3. Statement of intent

4. Relationships of the UK Renal Registry

5. The role of the UK Renal Registry for patients

6. The role of the UK Renal Registry for nephrologists

7. The role of the UK Renal Registry for Trust managers

8. The role of the UK Renal Registry for commissioning agencies

9. The role of the UK Renal Registry in national quality assurance schemes

10. References and websites

\section{A:1 Executive summary}

1.1 The UK Renal Registry (UKRR) was established by the Renal Association to act as a resource in the development of patient care in renal disease.

1.2 The Registry acts as a source of comparative data for audit, benchmarking, planning, policy and research. The collection and analysis of sequential biochemical and haematological data is a unique feature of the UKRR.

1.3 The Renal Registry Data Set Specification (RRDSS) defines the data items that are required to be sent from participating renal centres for analysis by the UKRR.

1.4 Data is collected quarterly to maintain centre-level quality assurance, with the results being published in an annual report.

1.5 Activity is funded from commissioning agencies by a capitation fee on renal patients.
1.6 The UKRR is responsible, with the express agreement of participants, for providing data to Trusts, Primary Care Trusts (PCTs) until March 2013, commissioning authorities and the European Renal Association - European Dialysis and Transplant Association (ERA-EDTA) Registry.

1.7 The development of the Registry is open to influence from all interested parties, including clinicians, Trusts, commissioning authorities and patient groups.

1.8 The Registry is non-profit making and has a registered charitable status through the Renal Association.

\section{A:2 Introduction}

2.1 Registry-based national specialty comparative audit is one of the cornerstones of NHS development. The Renal National Service Framework (NSF), published in two sections in 2004 and 2005, recommended the participation of all renal centres in comparative audit through the UK Renal Registry, with co-temporaneous documents defining the necessary information strategies [1-4].

2.2 The Chief Executives of Trusts are responsible for clinical governance and audit will be an essential part of that agenda [5].

2.3 Demographic information on patients receiving renal replacement therapy (RRT) throughout Europe was collected from 1965 in the Registry of the ERA-EDTA. This voluntary exercise was conducted on paper and by post, demanded considerable effort and time from participating centres

\begin{tabular}{ll}
\hline KARGER & (C) 2013 S. Karger AG, Basel \\
Fax +4161306 12 34 & 1160-2110/13/1235-0195\$38.00/0 \\
$\begin{array}{l}\text { E-Mail karger@karger.ch } \\
\text { www.karger.com }\end{array}$ & $\begin{array}{l}\text { Accessible online at: } \\
\text { www.karger.com/nec }\end{array}$
\end{tabular}


and eventually proved impossible to sustain. Latterly, the incompleteness of UK data returns to the ERA-EDTA made it impossible to build a picture of the activity of RRT in the UK for planning and policy purposes. Subsequently, five ad hoc national data collections from England \& Wales were solicited from renal centres in 1992, 1996, 1999, 2002 and 2004 to fill this gap. The UKRR through its quarterly returns has established a system to place routine data collection and analysis on a permanent basis. The next stage is to progress the work to include information on chronic kidney disease.

2.4 Together with the need to know the demographic and structural elements, the NHS has developed a need to underpin clinical activity more rigorously through the scientific evidence base (for example, the Cochrane Initiative) and by quality assurance activity through audit. These initiatives require comprehensive information about the structures, processes and outcomes of RRT, which go well beyond the detail previously compiled by the ERA-EDTA.

2.5 The Registry is recognised as one of the very few high-quality clinical databases available for general use [6]. The collection of data by download of electronic records from routine clinical databases is uncommon, has been highly successful and is being imitated worldwide.

2.6 The Renal Association has made a start in the area of audit by publishing guidelines in renal 'Clinical Standards' documents. It was apparent during the development of the standards that many of the desirable criteria of clinical performance were uncertain or unknown and that only the accumulated data of practicing renal centres could provide the evidence for advice on best practice and what might be achievable. A common data registration provides the simplest device for such an exercise. The data currently gathered audits a proportion of the Renal Association standards, partly due to some data items required not being available in the dataset and partly due to data not being either completed in or extracted from renal systems. There therefore needs to be a review of the dataset and a drive for more complete data returns by renal centres.

2.7 It can be seen that the need for a Registry of RRT has developed for a variety of reasons: international comparisons, national planning, local Trust, PCT and health authority management, standard setting, audit and research. The opportunity for data gathering arises partly from improvements in information technology. Although it was possible to see the need for a national renal database 20 years ago, the circumstances have become ideal for the maintenance of a data repository, supported by the clinical users and resourced for national benchmarking as a routine part of RRT management.

2.8 The provisional expectations of earlier Annual Reports can now be replaced by confident assertions, built on the experience of fourteen years of publication, about the role and potential of the UKRR. The integration of the various elements of Renal Association strategy is being pursued through the Clinical Affairs Board (CAB).

\section{A:3 Statement of intent}

The Renal Registry provides a focus for the collection and analysis of standardised data relating to the incidence, clinical management and outcome of renal disease. Data will be accepted quarterly by automatic downloading from renal centre databases. There will be a core dataset, with optional elements of special interest that may be entered by agreement for defined periods. A report will be published annually to allow a comparative audit of facilities, patient demographics, quality of care and outcome measures. Reports using the data collected can be generated at centre, regional and national level by interested parties via the data portal on the UKRR website www.renalreg.com. Participation is mandated in England through the recommendation in the Renal National Service Framework. There has been an early concentration on RRT, including transplantation, with an intention to extend this to other nephrological activity in the near future. The Registry will provide an independent source of data and analysis on national activity in renal disease.

\section{A:4 Relationships of the UK Renal Registry}

4.1 The UK Renal Registry is a registered charity through the Renal Association (No. 2229663). It was established by a committee of the Renal Association, with additional representation from the British 
Transplantation Society, the British Association for Paediatric Nephrology, the Scottish Renal Registry, Wales and Northern Ireland. There is cross-representation with both the Renal Association Standards and Clinical Trials Committees and the Clinical Affairs Board. The Registry has a Chairman and Honorary Secretary nominated by the Renal Association. The Registry has an observer from the Department of Health, a participant from the National Kidney Federation (NKF) (patients' association), the Royal College of Nursing, the Association for Clinical Biochemistry and a member representing the Health Care Commissioners.

4.2 A number of sub-committees have been instituted as the database and renal centre participation developed, particularly for data analysis and interpretation for the Annual Report. Further specialised panels may be developed for publications and the dissemination of UKRR analyses.

4.3 The Scottish Renal Registry sends data to the UK Renal Registry for joint reporting and comparison.

4.4 The return of English, Welsh and Northern Ireland data to the EDTA-ERA Registry will be through the Renal Registry. The Scottish Renal Registry already sends data directly to the EDTA-ERA Registry.

4.5 A paediatric database has been developed in collaboration with the UKRR, and the two databases are compatible. These two databases are in the process of being integrated, which will allow long-term studies of renal cohorts over a wide age range.

4.6 Close collaboration has been achieved with the NHS Blood and Transplant organisation - (formerly UK Transplant) giving joint benefits. Data aggregation and integration has led to joint presentations and publications. The description of the entire patient journey in RRT by this means is a source of continuing insight and usefulness.

4.7 The basis of participation for renal centres nationally is an agreement to accept the RRDSS for the transmission and retention of data. This is currently increasing to a core dataset of approximately 400 items and further optional elements, which will be returned on a special understanding with the renal centres for a defined period of reporting.

4.8 The retention of patient identifiable information, necessary in particular for the adequate tracing of patients, has been approved by the National Information Governance Board (NIGB) This is renewed on an annual basis along with audit of the information governance arrangements within the Registry through completion of the Connecting for Health Toolkit.

\section{A:5 The role of the UK Renal Registry for patients}

5.1 The goal of the UKRR is to improve care for patients with renal disease. The appropriate use of UKRR information should improve equity of access to care, adequacy of facilities, availability of important but high-cost therapies and the efficient use of resources. The continuing comparative audit of the quality of care should facilitate the improvement of care and outcomes of care.

5.2 A leaflet and poster has been provided, in collaboration with the NKF, by which patients may opt out of the collection of identifiable data by the UKRR if they wish. This was renewed in 2012 as part of the Renal Registry's NIGB submission, however opt out remains low.

5.3 Information from the UKRR will complement the individual records available on 'RenalPatientView' where it is accessible.

\section{A:6 The role of the UK Renal Registry for nephrologists}

6.1 The clinical community have become increasingly aware of the need to define and understand their activities, particularly in relation to national standards and in comparison with other renal centres.

6.2 The UKRR is run by a Committee of the Renal Association and therefore by colleagues with similar concerns and experience. The governance structure of the Registry will be reviewed by the Renal Association in 2013, this review will incorporate the structure and function of the Committee.

6.3 The Renal Standards documents are designed to give a basis for centre structure and performance, as well as patient-based elements such as case mix and outcomes. It is anticipated that Standards will become increasingly based on research evidence.

6.4 The UKRR data are available to allow the comparative review of many elements of renal centre practice. Centre data are presented to allow a contrast of individual centre activity and results 
against national aggregated data. Sophisticated analyses of patient survival for example, are a unique resource to exclude any anomalies of performance and standardise for centre caseload, etc.

6.5 Reports of demographic and treatment variables are available to the participating centres for distribution to Trusts, PCTs, Strategic Health Authorities and Commissioners, as well as renal networks, as required and agreed with the centre. Reports should facilitate discussion between clinicians, Trust officers and commissioners.

6.6 The Registry welcome suggestions for topics of national audit or research that colleagues feel are of sufficiently widespread interest for the UKRR to undertake.

6.7 The database has been designed to provide research facilities and for future participation in national and international trials. Members of the Renal Association and other interested parties are welcome to apply to the Registry Study Groups to conduct local or national audit and research using the database. All such projects will need the agreement of the Registry Study Groups and any costs involved may need to be met by the applicants.

6.8 These facilities will be sustainable only through cooperation between nephrologists and the UKRR. There is a need for high-quality and comprehensive data entry at source.,

6.9 Centres will need to develop an 'annual informatics plan', to review the maintenance and improvement of data collection organisation and returns to the UKRR. This will help maintain the accuracy, timeliness and completeness of clinical data and also in parallel, support the career development of informatics staff.

\section{A:7 The role of the UK Renal Registry for Trust managers}

7.1 As the basis of the clinical governance initiative, the gathering and presentation of clinical data are regarded as essential parts of routine patient management in the health service.

7.2 One of the principles of health service informatics is that the best data are acquired from clinical information recorded at the point of health care delivery.
7.3 Renal services data entered on local systems by staff directly engaged with patients are likely to be of the highest quality and it is these that the UKRR intends to capture.

7.4 The UKRR provides a cost-effective source of detailed information on renal services.

7.5 The regular reports of the UKRR supply details of patient demographics, treatment numbers, treatment quality and outcomes. Data are compared with both national standards and national performance, for benchmarking and quality assurance. The assessment of contract activity and service delivery is possible through these data returns, without the need for further costly Trust or commissioner administrative activity. These data should be particularly valuable to contracts managers and those responsible for clinical governance.

7.6 Data are available on centre case mix, infrastructure and facilities.

7.7 Work is progressing on the data capture and analysis from patients with renal disease other than those requiring RRT and will become available in time (e.g. CKD).

\section{A:8 The role of the UK Renal Registry for Commissioners of health care}

8.1 The commissioning of healthcare within England is evolving again providing uncertainty around the arrangements. However, from meetings with commissioners it is apparent however the powerful role accurate data plays in their decisions.

8.2 The use of information sources such as the UKRR is advised in the National Renal Review in order to promote benchmarking and quality assurance of renal programmes. The comprehensive tracking of relatively small but costly renal cohorts should be regarded as a routine part of speciality case management.

8.3 The UKRR provides validated, comparative reports of renal centre activity on a regular basis to participating centres. These allow assessment of centre performance across a wide range of variables relating to structure, process and outcome measures.

8.4 There are economies of scale in the performance of audit through the UKRR, since multiple local audits are not required. 
8.5 The incidence of RRT treated locally, mortality and renal transplant rates should also be of interest. The assessment of referral and treatment patterns of patients with established (end stage) renal failure by postcode analysis indicates the geographical origin. This information also allows the expression of differences relating to geography, ethnicity and social deprivation. These data may also identify potential unmet need in the population and permit assessment on the equity of service provision. In the future, the UKRR database should also provide information on nephrology and pre-dialysis patients (CKD). This will allow a prediction of the need for RRT facilities, as well as indicating the opportunities for beneficial intervention.

8.6 UKRR data are used to track patient acceptance and prevalence rates over time, which allows the modelling of future demand and the validation of these predictions.

8.7 Information on the clinical diagnosis of new and existing RRT patients may help identify areas where possible preventive measures may have maximal effect.

8.8 The higher acceptance rates in the elderly, and the increasing demand from ethnic groups due to a high prevalence of renal, circulatory and diabetic disease, are measurable.

8.9 Comparative data are available in all categories for national and regional benchmarking.
8.10 The UKRR offers independent expertise in the analysis of renal services data and their interpretation, a resource that is widely required but difficult to otherwise obtain.

8.11 In 2013 cost of supporting the UKRR will be $\mathfrak{E} 19$ per registered RRT patient per annum (no change from 2012), which is less than $0.05 \%$ of the typical cost of a dialysis patient per annum. It is expected that this cost will need to be made explicit within the renal services contract.

\section{A:9 The role of the UK Renal Registry for national quality assurance agencies}

9.1 The role of the UKRR in the national quality assurance programme of the Healthcare Quality Improvement Partnership, will depend on the decisions on the role and responsibilities of that agency and their means to discharging them.

9.2 The demographic, diagnostic and outcomes data could support the investigation of clinical effectiveness.

9.3 The case mix information and comorbidity data that would allow better assessment of survival statistics remains incomplete. There is also some clinical scepticism whether 'correction' of outcome data would reflect the realities of clinical practice.

\section{A:10 References}

1 http://www.kidney.org.uk/campaigns/Renal-nsf/pt1-nsf-content-report.pdf 2 http://www.kidney.org.uk/campaigns/Renal-nsf/nsf-pt2.pdf

3 RNSF IS 1 http://www.dh.gov.uk/assetRoot/04/07/79/25/04077925.pdf

4 RNSF IS 2 http://www.dh.gov.uk/assetRoot/04/11/35/05/04113505.pdf
Black N. Clinical governance: fine words or action? Br Med J 1998;316: 297-8

6 Black N. High-quality clinical databases: breaking down barriers [Editorial]. Lancet 1999;353:1205-6 